\title{
Analysis of immune status after iodine- 125 permanent brachytherapy in prostate cancer
}

This article was published in the following Dove Press journal:

OncoTargets and Therapy

15 May 2017

Number of times this article has been viewed

E Du

Lin Wang

Chang-ying Li

Chang-wen Zhang

Yan-chun Qu

Ran-lu Liu

Yong $\mathrm{Xu}$

Kuo Yang

Zhi-hong Zhang

Tianjin Institute of Urology, the 2nd Hospital of Tianjin Medical University, Tianjin, People's Republic of China
Correspondence: Kuo Yang;

Zhi-hong Zhang

Tianjin Institute of Urology, the 2nd

Hospital of Tianjin Medical University,

23 Pingjiang Road, Hexi Distribute,

Tianjin 3002II, People's Republic

of China

Tel +862288329296

Email ykuoster@126.com;

zhangzhihongtianj@I63.com
Background: Permanent prostate brachytherapy (PPB) is an effective treatment choice for low and intermediate risk prostate cancer (PCa). However, the impact of PPB on tumor immune status is still poorly understood. This study aimed to assess the immune status in PCa patients before and at different time points after PPB (1, 3, 6, and 12 months).

Methods: Blood was collected from 32 patients with low and intermediate risk PCa and 12 healthy volunteers. The frequency of immunocompetent cells was identified by flow cytometry. The concentration of immunoglobulins and complements was detected by ELISA.

Results: Various immunocompetent cells were dysregulated in PCa patients compared with healthy volunteers. Peripheral serum prostate-specific antigen (PSA) decreased rapidly at the first month after PPB treatment, and the peripheral serum PSA became very low at 6 months after PPB treatment. CD3+ T cells, CD4+ T cells, CD3-CD16+/56+ natural killer (NK) cells were increased significantly at certain time points after PPB. Although the percentage of the CD8+ T cells did not change markedly, the ratio of CD4/CD8 increased significantly at 3 months after PPB $(P=0.0196)$. There was no influence of PPB on B cells number, but the concentration of immunoglobulins IgM, IgG, and IgA, and complements $\mathrm{C} 3$ and $\mathrm{C} 4$ in patients increased at some time points after PPB.

Conclusion: The immunocompetent cells are dysregulated in PCa patients. PPB treatment could effectively kill tumor cells and then stimulate cellular immunity and humoral immunity in PCa patients.

Keywords: prostate cancer, iodine-125, permanent prostate brachytherapy, immune status

\section{Introduction}

Prostate cancer (PCa) is one of the most common malignancies of urological system. It is the second leading cause of cancer-specific deaths among men throughout the world. ${ }^{1}$ With the increasing use of peripheral serum prostate-specific antigen (PSA) screening, $\mathrm{PCa}$ incidence is rising rapidly worldwide in recent years. ${ }^{2}$ Most of the cases newly diagnosed are low- and intermediate-risk ones with low peripheral serum PSA level and Gleason score, who can take permanent prostate brachytherapy (PPB) with radioactive seeds iodine-125 (I-125) as a treatment choice and obtain similar outcome as radical prostatectomy or external radiation therapy (XRT).

The presence of an immune response to $\mathrm{PCa}$ can be seen in the form of tumor-infiltrating lymphocytes, particularly CD8+ T cells, which have been shown to be a positive prognostic factor in $\mathrm{PCa}$ and others..$^{3-9}$ As a valuable treatment choice for low- and intermediate-risk PCa, PPB gradually destroys tumor tissue by killing the tumor cells, leaving tumor proteins and tumor-associated antigens intact, which can potentially modulate the host's immune response. However, the impact of PPB on tumor immune status is still poorly understood. In this perspective, the study was 
performed to assess the immune status after PPB in $\mathrm{PCa}$ patients, which may be helpful to reveal immunologic mechanisms of clinical outcomes after PPB therapy.

\section{Materials and methods Patient characteristics}

Thirty-two patients with low- and intermediate-risk PCa and 12 healthy volunteers were enrolled in this study from January 2010 to June 2015 in the second hospital of Tianjin Medical University. All patients were evaluated with the International Prostate Symptom Score (IPSS) questionnaire assessment score as previously. ${ }^{10}$ Subjects were diagnosed with presurgery biopsies separately by two senior pathologists. All patients were free of chemo or endocrinological therapy before PPB and had not suffered from any other type of tumor. Age, Gleason score, PSA, and transrectal ultrasonography (TRUS)-based prostate volume were collected, respectively, for all patients. Peripheral serum PSA was obtained at different time points after PPB treatment (1, 3, 6 , and 12 months). Written informed consent was obtained from all patients and healthy volunteers. The study was approved by the ethical committee of Tianjin Medical University. The patient characteristics and treatment outcome are summarized in Table 1.

\section{Permanent prostate brachytherapy treatment}

Briefly, the patient had a general anesthesia and PPB was performed by using the "real-time" intraoperative planning method guided by TRUS. The radioactive seeds were inserted transperineally according to a modified peripherally loaded

Table I Patient characteristics and treatment outcome

\begin{tabular}{ll}
\hline Characteristics & Mean (\%) \\
\hline Gleason score & \\
5 & $7 / 32(21.8)$ \\
6 & $11 / 32(34.4)$ \\
7 & $14 / 32(43.7)$ \\
Pathological stage & \\
TI & $15 / 32(46.9)$ \\
T2 & $17 / 32(53.1)$ \\
\hline & Average \\
& (min-max) \\
\hline Preoperative PSA (ng/mL) & $8.64(4.5-12.0)$ \\
Mean IPSS & $12.13(8-20)$ \\
Prostate volume (mL) & $52.6(42.7-65.0)$ \\
Age (years) & \\
Control & $56(39-68)$ \\
PCa & $69(48-83)$ \\
\hline
\end{tabular}

Abbreviations: IPSS, International Prostate Symptom Score; PCa, prostate cancer; PSA, prostate-specific antigen.
Seattle technique 17. I-125 was used for all implants with a mean seed activity of $0.45 \mathrm{mCi}$ per seed. All procedures were completed by a single surgeon. All patients were given $\alpha$-blockers the day after brachytherapy for a minimum of 1 week and continued taking them for as long as deemed necessary by the physician. Computerized tomography-based, dose evaluation was performed for all patients at 1 month after PPB as before. ${ }^{10}$

\section{Flow cytometry analysis}

A volume of $200 \mu \mathrm{L}$ blood was labeled with $20 \mu \mathrm{L}$ fluorescence-conjugated antihuman antibodies (double staining with CD3 FITC/CD4 PE, CD3 FITC/CD8 PE, CD3 FITC/ CD19 PE, CD3 FITC/CD16/CD56 PE, BD Pharmingen, San Diego, CA, USA) for 30 minutes following the manufacturer's instructions and recommendations. Then the red blood cells were lysed, washed 3 times in phosphate-buffered saline (PBS), and then fixed in 4\% paraformaldehyde in PBS, and cytometry was performed by using Cell Quest software on a FACScan flow cytometer (Becton Dickinson, Mountain View, CA, USA). Suitable negative isotype controls were used to rule out the background fluorescence. The data were generated by cytofluorometric analyses of 10,000 events. The positive population for each marker was determined using quadrant statistics. The data were analyzed using FlowJo 7.6 software.

\section{ELISA}

Detection of $\operatorname{IgA}$, IgM, IgG, C3, and C4 in serum at different time points in PCa patients was performed with human ELISA kit (Mabtech, Nacka Strand, Sweden). In brief, 96-well microtiter plates were coated with capture antibodies, incubated overnight at $4^{\circ} \mathrm{C}$, and blocked for 1 hour. After washing, $100 \mu \mathrm{L}$ of serum was added to each well and incubated for 2 hours. Bound cytokines were detected using biotinylated mouse antihuman IgA, IgM, IgG, C3, and $\mathrm{C} 4$, and subsequently avidin-horseradish peroxidase. Color was developed with TMB substrate solution. Reaction was terminated with $\mathrm{NaOH}$ solutions, and absorbance was measured at $450 \mathrm{~nm}$ using a microplate reader (Molecular Devices, Sunnyvale, CA, USA).

\section{Follow-up and statistical analysis}

I-125 has a half-life of 60 days, and significant radioactive decay will occur over 1 year after implant with most of the decay occurring in the first 6 months. Follow-up was scheduled at 1, 3, 6, and 12 months after PPB. These patients were followed up to assess changes in PSA values, frequency of 
immunocompetent cells, and concentration of immunoglobulins and complement.

The results represented data range and were expressed in a box-plot format by GraphPad Prism 5. Quantitative variables were analyzed by variance analysis with Student's $t$-test. The case with missing data was excluded from further analysis. The significant threshold was set at $P \leq 0.05$. All statistics were conducted with SPSS 18.0 software package (IBM Corp., Armonk, NY, USA).

\section{Results}

\section{Various immunocompetent cells were dysregulated in PCa patients}

Immunocompetent cells including T cells, B cells, and NK cells play an important role in antitumor immune response and are closely associated with the development of PCa. PBMCs obtained from PCa patients and healthy volunteers were stained in $\mathrm{T}$ cell subsets (CD3+, CD4+ helper, and CD8+ cytotoxic), B cells (CD19+), and NK cells (CD3- CD16+/CD56+). The percentage of CD3+ T, CD4+ T, CD8+ T (Figure 1A-C), and CD3-CD16+/CD56+ NK cells (Figure 1E) was much lower in the PCa patient population than in the healthy volunteers, and the ratio of CD4/CD8 (Figure 1D) was also significantly reduced in the patient population. However, there was no significant difference between the two groups in CD19+ B cells (Figure 1F).

\section{The impact of PPB treatment on the peripheral serum PSA}

Peripheral serum PSA was taken as a golden standard for the assessment of impacts by different treatment strategy for PCa patients. The peripheral serum PSA decreased rapidly at 1 month after PPB treatment, and the peripheral serum PSA became very low at 6 months after PPB treatment. Then, the peripheral serum PSA reached a new nadir balance level, which may be consistent with the half-life of I-125 seed. The details are outlined in Figure 2. The observed complications were mostly minor, with $20 \%$ (4/32) reporting a mild hematuria and 15\% (3/32) reporting perineal/scrotum hematoma with no need for further treatment. Rectal injury and urinary incontinence, which was defined as the need for one or more pads per day, were not observed.

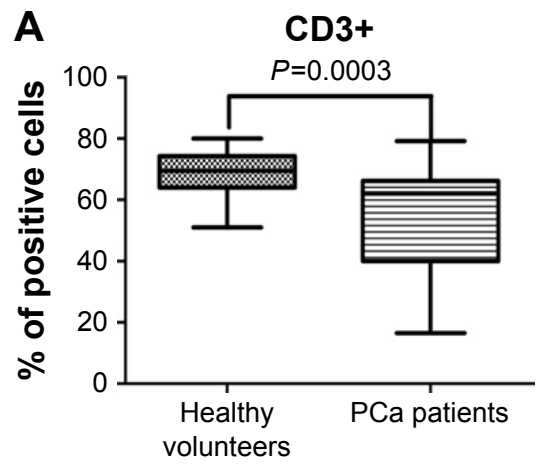

D

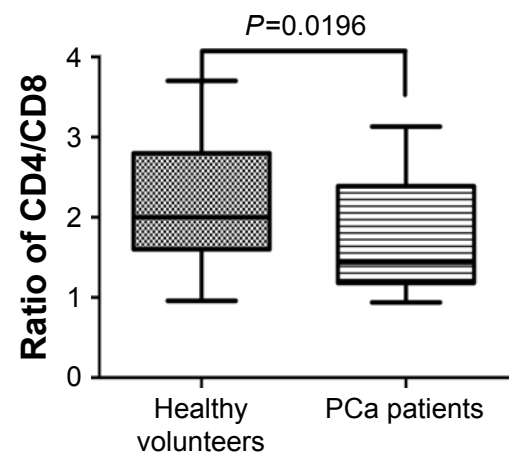

B

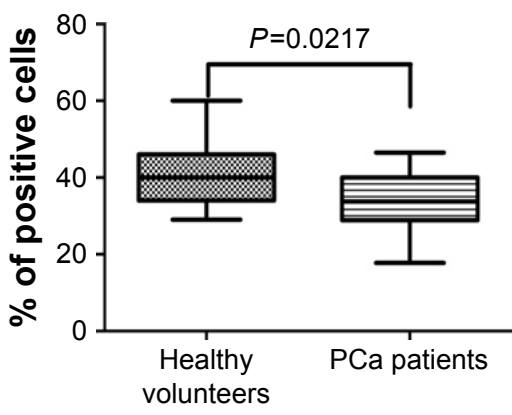

E

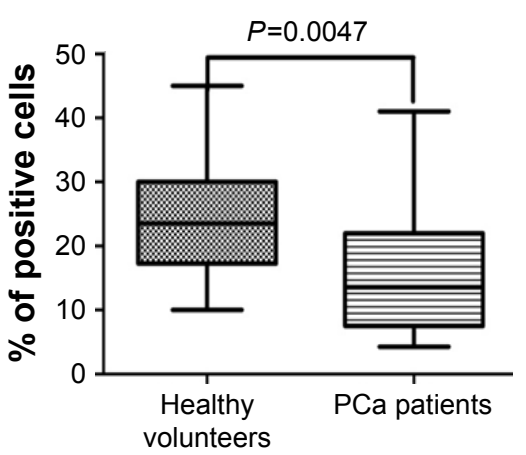

C

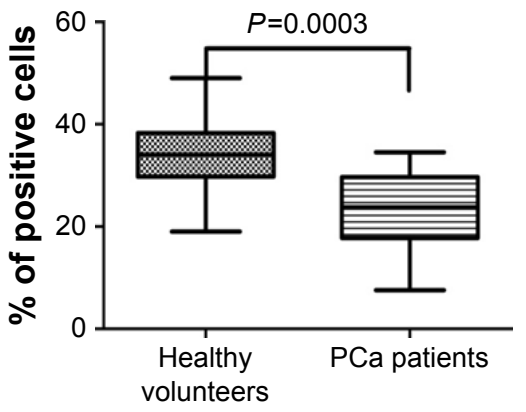

$\mathbf{F}$

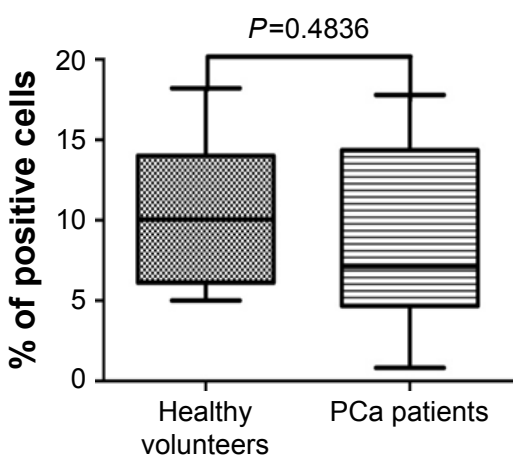

Figure I Flow cytometric results of various immunocompetent cells in PCa patients and healthy volunteers.

Notes: Phenotypic studies were performed by flow cytometric analysis using fluorescence-conjugated antihuman antibodies double-staining CD3 FITC/CD4 PE, CD3 FITC/ CD8 PE, CD3 FITC/CDI9 PE, CD3 FITC/CDI6/CD56 PE. The data were expressed in a box-plot format by GraphPad Prism 5. Various immunocompetent cells including $\mathrm{CD} 3+\mathrm{T}(\mathbf{A}), \mathrm{CD} 4+\mathrm{T}(\mathbf{B}), \mathrm{CD} 8+\mathrm{T}(\mathbf{C})$, and CD3-CD 16+/CD56+ (E) were dysregulated in PCa patients, and the ratio of CD4/CD8 (D) was also significantly reduced in the patient population. However, there was no significant difference between the two groups in CDI9+ B cells $(\mathbf{F})$.

Abbreviation: PCa, prostate cancer. 


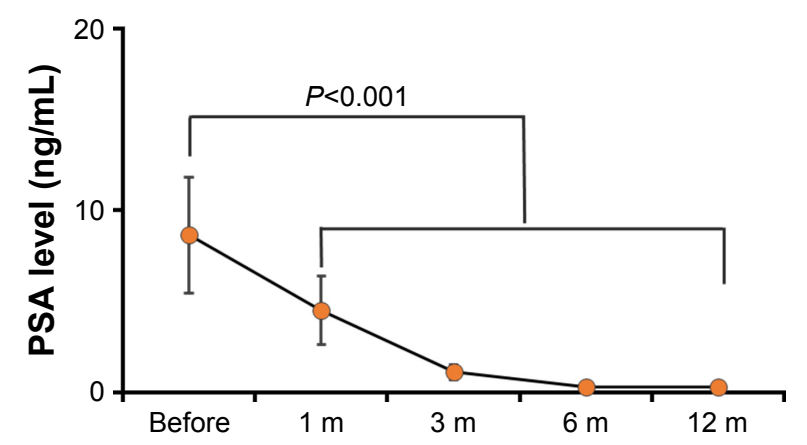

Figure 2 The peripheral serum PSA level before and at different time points after PPB treatment.

Note: The peripheral serum PSA decreased rapidly at the first month after PPB treatment, and the peripheral serum PSA became very low at 6 months after PPB treatment.

Abbreviations: PPB, permanent prostate brachytherapy; PSA, prostate-specific antigen.

\section{The effects of PPB on cellular immunity}

To evaluate the effects of PPB on cellular immunity, we assayed the immunocompetent cells before and at different time points after PPB treatment, and the results showed that the percentage of the $\mathrm{CD} 3+\mathrm{T}$ and $\mathrm{CD} 4+\mathrm{T}$ cells in $\mathrm{PCa}$ patients was increased significantly at 3 months after PPB. Although the percentage of the CD8+ T cells did not change markedly, the ratio of $\mathrm{CD} 4 / \mathrm{CD} 8$ was increased significantly
( $P=0.0335$ ). Moreover, CD3-CD16+/CD56+ NK cells were increased significantly at 12 months after PPB. However, the $B$ cells were decreased significantly at 3 months after PPB ( $P=0.041$; Figure 3).

\section{The effects of PPB on humoral immunity}

There was no influence of PPB on B cells number, and immunoglobulin was a sensitive indicator reflecting the function of B-cell immune responses. To further confirm the effects of PPB on humoral immunity, we tested the immunoglobulins IgM, IgG, and IgA, and complements $\mathrm{C} 3$ and $\mathrm{C} 4$ before and at different time points after PPB treatment (Figure 4). The results manifested that the IgM concentration in patients was significantly increased at 1, 3, and 12 months after PPB; the $\mathrm{IgG}$ concentration was significantly increased at 3 months after PPB; the $\mathrm{C} 3$ concentration was significantly increased at 1, 3, and 12 months after PPB; the C4 concentration was significantly increased at 1 month after PPB. Meanwhile, the IgA concentration was also increased at 1 month after PPB, but the difference did not reach statistical significance.

\section{Discussion}

PPB is an effective radical treatment for low- and intermediaterisk $\mathrm{PCa} .{ }^{1,2}$ Recently, this minimally invasive treatment is also

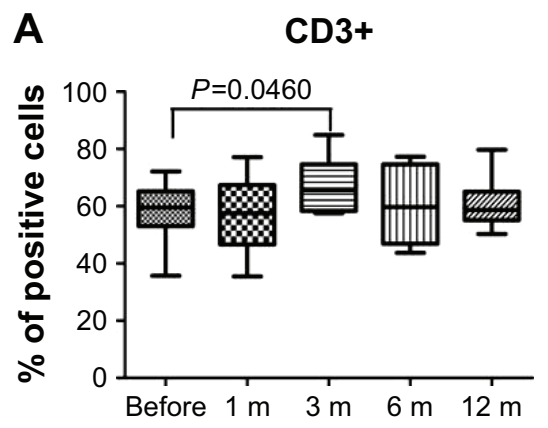

D

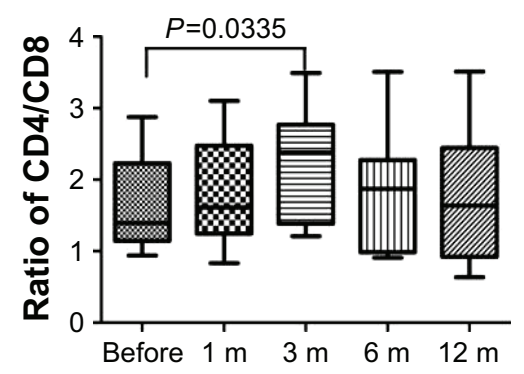

B

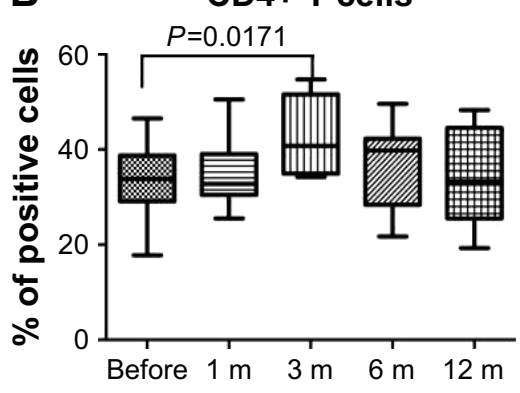

E

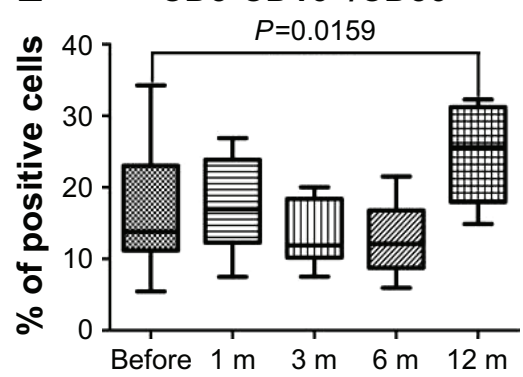

C

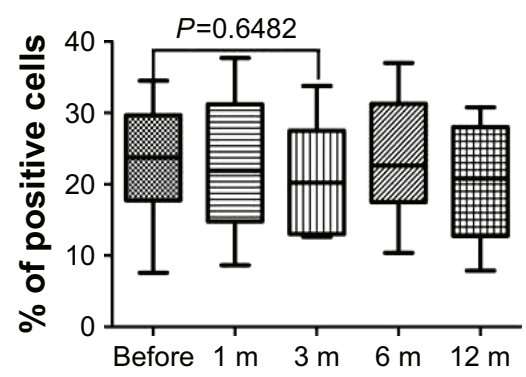

$\mathbf{F}$

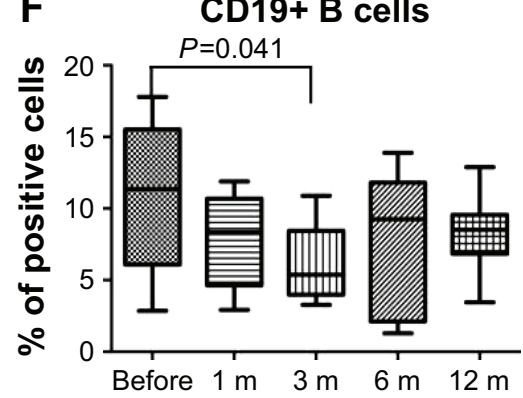

Figure 3 Flow cytometric results of the frequency of immunocompetent cells before and after PPB

Notes: Phenotypic studies were performed by flow cytometric analysis using fluorescence-conjugated antihuman antibodies double staining CD3 FITC/CD4 PE, CD3 FITC/ CD8 PE, CD3 FITC/CDI9 PE, CD3 FITC/CDI6/CD56 PE. The results represented data range and were expressed in a box-plot format by GraphPad Prism 5. CD3+ T cells (A), CD4+ T cells (B), ratio of CD4/CD8 (D), and CD3-CDI6+/CD56+ NK cells (E) were increased at some time point after PPB, but CD8+ T (C) cells did not change markedly. Moreover, B cells (F) decreased significantly at 3 months after PPB.

Abbreviation: PPB, permanent prostate brachytherapy. 

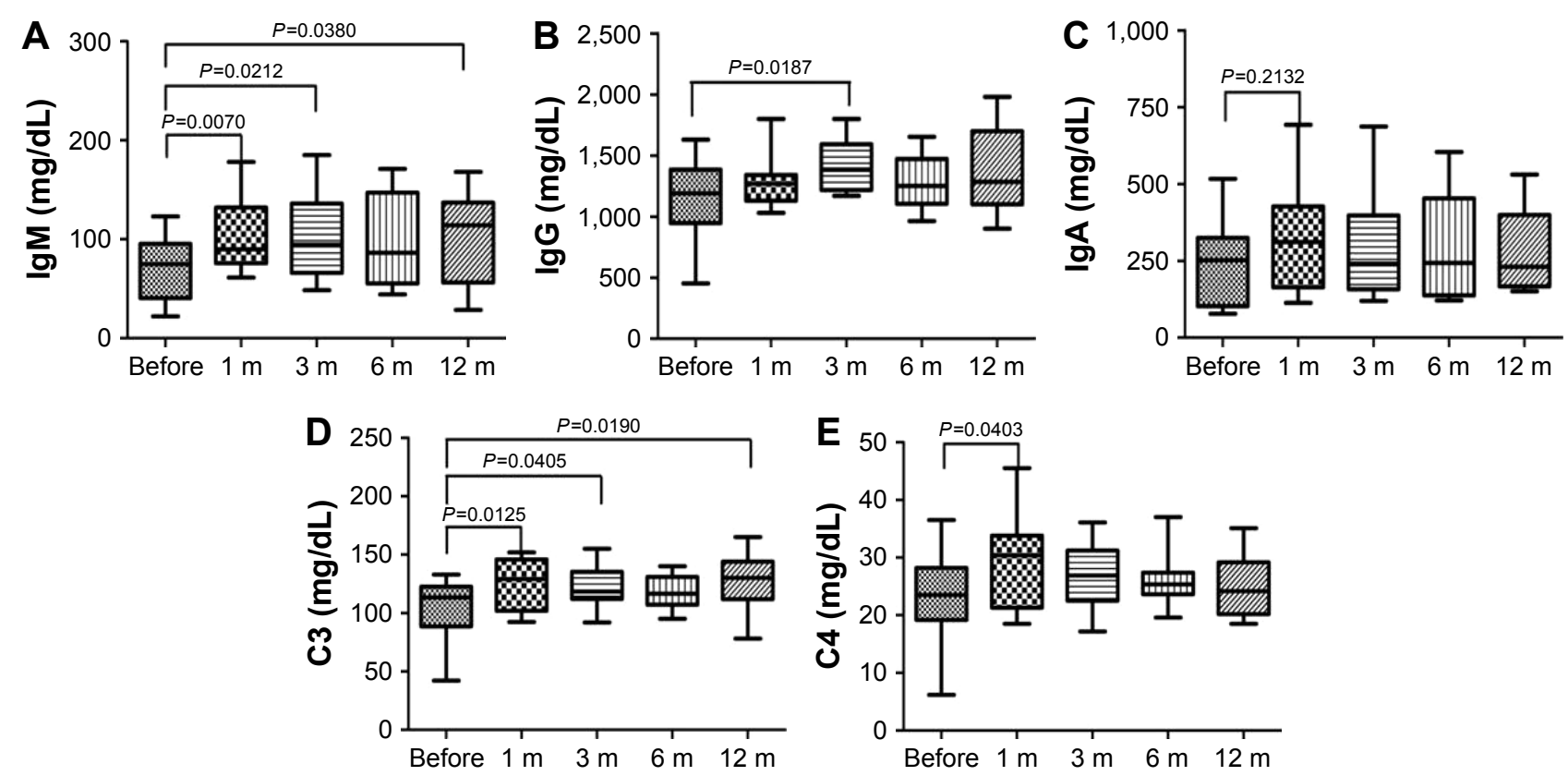

Figure 4 ELISA results of the concentrations of immunoglobulins and complements before and after PPB.

Notes: The results represented data range and were expressed in a box-plot format by GraphPad Prism 5 . The concentrations of IgM (A), IgG (B), C3 (D), and C4 (E) showed significant increase at some time points. The $\operatorname{lgA}(\mathbf{C})$ concentration was also increased at I month after PPB, but the difference did not reach statistical significance. Abbreviation: PPB, permanent prostate brachytherapy.

becoming more widely used for Chinese PCa patients and obtains similar outcomes as radical prostatectomy or XRT.

The development and progression of $\mathrm{PCa}$ was closely associated with the special immune condition or environment of these patients. The ability to suppress cancer by innate and adaptive immunity has been collectively termed cancer immunosurveillance. ${ }^{11,12}$ The deficiency in prostate-associated lymphocytes enables a tumor to escape from surveillance on the part of the immune system. ${ }^{13}$ The prostate is part of the mucosal immune system: intraepithelial region consists of CD3+ T cells, predominantly CD8+, as well as NK, DC, and B cells; the lymphoid aggregates form below the epithelial layer, arranged as B-cell follicles, with parafollicular areas composed of mostly CD4+ T cells and DCs. ${ }^{14} \mathrm{~T}$ cells provide protective immunity against tumor antigens by generating effector cells, which cooperate to eliminate antigens and memory cells, which can survive for long periods with enhanced abilities to control recurring tumor antigens. Following activation, CD8+ T cells that are usually associated with cytotoxic functions can migrate to sites of tumor and kill tumor cells, whereas CD4+ T cells contribute to the elimination of pathogens by trafficking to tumor tissues and providing help to innate immune responses, B cells, as well as CD8+ T cells. The balance between CD4+ T and CD8+ T cells maintains the stability of immune homeostasis. A renewed interest has emerged about the usefulness of CD4/ CD8 ratio as a strong marker of immune activation, ${ }^{15}$ and the lower ratio of CD4/CD8 means the lower immunocyte response to tumor. This study indicated that the frequency of peripheral T cells and NK cells, and the ratio of CD4/CD8 were much lower in the PCa patients, which was consistent with other tumors. ${ }^{7,14,16}$

Peripheral serum PSA was taken as a widely used marker for assessing therapeutic response of PCa patients. I-125 has a half-life of 60 days and significant radioactive decay will occur over 1 year after implant with most of the decay occurring in the first 6 months. Therefore, we chose 1, 3, 6, and 12 months after PPB treatment as the time points. The peripheral serum PSA decreased rapidly at the first month, and the peripheral serum PSA became very low at 6 months after PPB treatment, which was consistent with the release velocity of I-125 seed. Meanwhile, the results suggested that PPB is an effective radical treatment for low- and intermediate-risk $\mathrm{PCa}$ patients.

How might tumor antigens become immunogenic during tumor treatments? Cancer radiotherapy causes a direct cytotoxic effect on tumor cells; dying tumor cells release intracellular contents and proinflammatory cytokines that initiate the most potent antigen-presenting dendritic cells and attract granulocytes and/or macrophages. ${ }^{17-19}$ Despite these intriguing findings, there is little information available concerning the effect of PPB treatments on tumor immunity in PCa patients and the potential influence on clinical outcomes. To evaluate the effects of PPB on cellular 
immunity, we examined the immunocompetent cells before and at different time points after PPB treatment. The results showed that the percentages of the CD3+ T, CD4+ T, and NK cells in PCa patients were increased significantly at different times after PPB, which was consistent with the study of Nesslinger et al, in which they demonstrated that radiation therapy and hormone therapy induce antigen-specific immune responses in PCa patients as opposed to control patients treated with radical prostatectomy. ${ }^{3}$ Although the percentage of the CD8+ T cells did not change markedly, the ratio of CD4/CD8 was increased significantly ( $P=0.0196)$. CD8+ T cells and NK cells were both decreased at 3 months after PPB, we therefore hypothesized that some cytokines released after $\mathrm{PPB}$ attract $\mathrm{CD} 8+\mathrm{T}$ cells and NK cells into the tumor microenvironment and triggered the cellular immunity simultaneously. To prove this hypothesis, further studies of the molecular mechanisms are necessary to investigate the function and interactions of immunocompetent cells after PPB. There was also some divergent evidence. Some studies suggested radiation therapy activates CD8+ cytotoxic response via induction of a wide spectrum of inflammatory cytokine production, including MHC molecules, B7 and other co-stimulatory molecules, adhesion molecules, death receptors and heat shock proteins in tumor cells, stroma, and vascular endothelium. ${ }^{20,21}$ However, we failed to find significant change in CD8+ after PPB treatment. This divergence may be due to lower dose rate and longer duration of radiation on the prostate in the PPB patients compared with EXT patients.

Although tumor immune effect in general is given priority to cellular immunity, accumulating evidence is pointing to a role for $\mathrm{B}$ cells in modulating the immune response. $B$ cells were decreased significantly at 3 months after PPB. To further verify the effects of PPB on humoral immunity, we tested the immunoglobulins $\operatorname{IgM}, \operatorname{IgG}$, and $\mathrm{IgA}$, and complements including $\mathrm{C} 3$ and $\mathrm{C} 4$, which were sensitive indicators reflecting the function of $\mathrm{B}$ cell. The results manifested that the $\operatorname{IgM}, \operatorname{IgG}, \mathrm{C} 3$, and $\mathrm{C} 4$ concentration in patients was significantly increased at different time points after PPB. Meanwhile, the IgA concentration was increased at 1 month after PPB, but the difference was not statistically significant. Instead of PPB, ADT in humans can also induce effector-cell response to stimulation, and the generation of a prostate tissue-associated IgG antibody response. ${ }^{19}$ The present results suggested that PPB treatment can effectively activate the function of $B$ cells.

According to our knowledge, this is a pilot study focusing on the exploration of fluctuation rules of antitumor immune response and immune cells by PPB treatment of PCa. There were also some limits in our study. Firstly, only 32 patients who accepted PPB as a final treatment enrolled in this study, which is not enough for statistic power to reveal significant change after PPB treatment. Secondly, we followed up the fluctuation of the antitumor immune response only 1 year after PPB treatment. It was not enough to monitor the longterm consequences of PPB treatment on tumor immune response of $\mathrm{PCa}$ patients.

\section{Conclusion}

The immunocompetent cells were dysregulated in PCa patients. Peripheral serum PSA was decreased rapidly at the first month after PPB treatment, which suggests that PPB is an effective radical treatment for low- and intermediate-risk PCa. Further, most tumor immunity-associated parameters including immunocompetent cells, immunoglobulins, and complements were changed in divergent trend after treatment. A better understanding of the immune status after PPB may be helpful to reveal immunologic mechanisms of clinical outcomes after PPB therapy.

\section{Acknowledgments}

This study was supported by National Natural Science Foundation grants of China (Nos 81400686 and 81301949), Tianjin Science and Technology Plan Projects of China (No 13RCGFSY19100), Application Base and Frontier Technology Project (No 15JCQNJC10400), and Natural Science Foundation of Tianjin (No 16JCYBJC26500).

\section{Disclosure}

The authors report no conflicts of interest in this work.

\section{References}

1. Siegel R, Ma J, Zou Z, Jemal A. Cancer statistics, 2014. CA Cancer J Clin. 2014;64(1):9-29.

2. Cuzick J, Thorat MA, Andriole G, et al. Prevention and early detection of prostate cancer. Lancet Oncol. 2014;15(11):E484-E492.

3. Nesslinger NJ, Sahota RA, Stone B, et al. Standard treatments induce antigen-specific immune responses in prostate cancer. Clin Cancer Res. 2007;13(5):1493-1502.

4. Morse MD, McNeel DG. Prostate cancer patients on androgen deprivation therapy develop persistent changes in adaptive immune responses. Hum Immunol. 2010;71(5):496-504.

5. Friedman EJ. Immune modulation by ionizing radiation and its implications for cancer immunotherapy. Curr Pharm Des. 2002;8(19): $1765-1780$.

6. McBride WH, Chiang CS, Olson JL, et al. A sense of danger from radiation. Radiat Res. 2004;162(1):1-19.

7. Bose A, Chakraborty T, Chakraborty K, Pal S, Baral R. Dysregulation in immune functions is reflected in tumor cell cytotoxicity by peripheral blood mononuclear cells from head and neck squamous cell carcinoma patients. Cancer Immun. 2008;8:10. 
8. Galon J, Costes A, Sanchez-Cabo F, et al. Type, density, and location of immune cells within human colorectal tumors predict clinical outcome. Science. 2006;313(5795):1960-1964.

9. Azimi F, Scolyer RA, Rumcheva P, et al. Tumor-infiltrating lymphocyte grade is an independent predictor of sentinel lymph node status and survival in patients with cutaneous melanoma. J Clin Oncol. 2012;30(21):2678-2683.

10. Liu R, Luo F, Zhang Z, Xu Y. Iodine-125 seed implantation and deferred transurethral resection of the prostate for patients with lower urinary tract symptoms and localized prostate cancer. Clin Genitourin Cancer. 2013;11(3):251-255.

11. O'Sullivan T, Saddawi-Konefka R, Vermi W, et al. Cancer immunoediting by the innate immune system in the absence of adaptive immunity. J Exp Med. 2012;209(10):1869-1882.

12. Shankaran V, Ikeda H, Bruce AT, et al. IFNgamma and lymphocytes prevent primary tumour development and shape tumour immunogenicity. Nature. 2001;410(6832):1107-1111.

13. Di Carlo E, D'Antuono T, Pompa P, et al. The lack of epithelial interleukin-7 and BAFF/BLyS gene expression in prostate cancer as a possible mechanism of tumor escape from immunosurveillance. Clin Cancer Res. 2009;15(9):2979-2987.

14. Si TG, Wang JP, Guo Z. Analysis of circulating regulatory T cells (CD4+CD25+CD127-) after cryosurgery in prostate cancer. Asian J Androl. 2013;15(4):461-465.
15. Bruno G, Saracino A, Monno L, Angarano G. The revival of an "old" marker: CD4/CD8 ratio. AIDS Rev. Epub 2017 Feb 9.

16. Shibuya TY, Kim S, Nguyen K, et al. Covalent linking of proteins and cytokines to suture: enhancing the immune response of head and neck cancer patients. Laryngoscope. 2003;113(11):1870-1884.

17. Floercken A, Takvorian A, Singh A, et al. Modulation of regulatory T cells and myeloid-derived suppressor cells by sorafenib and sunitinib in renal cell carcinoma patients. J Clin Oncol. 2009;27(15).

18. Degl'Innocenti E, Grioni M, Capuano G, et al. Peripheral T-cell tolerance associated with prostate cancer is independent from CD4(+) CD25(+) regulatory T cells. Cancer Res. 2008;68(1):292-300.

19. Sorrentino C, Musiani P, Pompa P, Cipollone G, Di Carlo E. Androgen deprivation boosts prostatic infiltration of cytotoxic and regulatory $\mathrm{T}$ lymphocytes and has no effect on disease-free survival in prostate cancer patients. Clin Cancer Res. 2011;17(6):1571-1581.

20. Bronte V, Kasic T, Gri G, et al. Boosting antitumor responses of T lymphocytes infiltrating human prostate cancers. J Exp Med. 2005; 201(8):1257-1268.

21. Karja V, Aaltomaa S, Lipponen P, Isotalo T, Talja M, Mokka R. Tumour-infiltrating lymphocytes: A prognostic factor of PSA-free survival in patients with local prostate carcinoma treated by radical prostatectomy. Anticancer Res. 2005;25(6C):4435-4438.

\section{Publish your work in this journal}

OncoTargets and Therapy is an international, peer-reviewed, open access journal focusing on the pathological basis of all cancers, potential targets for therapy and treatment protocols employed to improve the management of cancer patients. The journal also focuses on the impact of management programs and new therapeutic agents and protocols on

\section{Dovepress}

patient perspectives such as quality of life, adherence and satisfaction. The manuscript management system is completely online and includes a very quick and fair peer-review system, which is all easy to use. Visit http://www.dovepress.com/testimonials.php to read real quotes from published authors. 